\title{
Subunits of ARID1 serve as novel biomarkers for the sensitivity to immune checkpoint inhibitors and prognosis of advanced non-small cell lung cancer
}

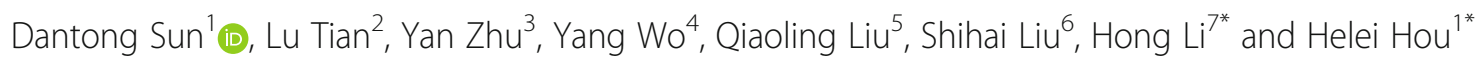

\begin{abstract}
Introduction: Patients with advanced non-small cell lung cancer (NSCLC) benefit from treatment with immune checkpoint inhibitors (ICls). Biomarkers such as programmed death-ligand 1 (PD-L1), the tumor mutational burden (TMB) and the mismatch repair (MMR) status are used to predict the prognosis of ICls therapy. Nevertheless, novel biomarkers need to be further investigated, and a systematic prognostic model is needed for the evaluation of the survival risks of ICls treatment.

Methods: A cohort of 240 patients who received ICls from the cBioPortal for Cancer Genomics was evaluated in this research. Clinical information and targeted sequencing data were acquired for analyses. The Kaplan-Meier plot method was used to perform survival analyses, and selected variables were then confirmed by a novel nomogram constructed by the "rms" package of R software.

Results: Seven percent of the NSCLC patients harbored ARIDIA mutations, while 4\% of the NSCLC patients harbored $A R I D 1 B$ mutations. Mutations in ARIDIA and ARIDIB were confirmed to be associated with sensitivity to ICls. Patients harboring these mutations were found to have a better response to treatment (ARID1A: $P=0.045 ; A R I D 1 B: P=0.034$ ) and prolonged progression-free survival (ARID1B: $P=0.032$ ). Here, a novel nomogram was constructed to predict the prognosis of ICls treatment. Elevation of the TMB, enhanced expression of PD-L1 and activation of the antigen presentation process and cellular immunity were found to be correlated with ARIDIA and ARIDIB mutations.
\end{abstract}

Conclusion: ARID1A and ARID1B could serve as novel biomarkers for the prognosis and sensitivity to ICls of advanced NSCLC.

Keywords: ARID1A, ARID1B, NSCLC, Immune checkpoint inhibitors, Prognosis

\footnotetext{
* Correspondence: hongli@shsci.org; houhelei@qdu.edu.cn

${ }^{7}$ State Key Laboratory of Oncogenes and Related Genes, Shanghai Cancer Institute, Renji Hospital, Shanghai Jiaotong University School of Medicine, Shanghai 200032, China

'Precision Medicine Center of Oncology, the Affiliated Hospital of Qingdao

University, 59 Haier Road, Qingdao 266000, Shandong, China

Full list of author information is available at the end of the article
}

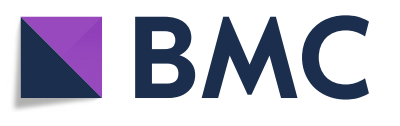

(c) The Author(s). 2020 Open Access This article is licensed under a Creative Commons Attribution 4.0 International License, which permits use, sharing, adaptation, distribution and reproduction in any medium or format, as long as you give appropriate credit to the original author(s) and the source, provide a link to the Creative Commons licence, and indicate if changes were made. The images or other third party material in this article are included in the article's Creative Commons licence, unless indicated otherwise in a credit line to the material. If material is not included in the article's Creative Commons licence and your intended use is not permitted by statutory regulation or exceeds the permitted use, you will need to obtain permission directly from the copyright holder. To view a copy of this licence, visit http://creativecommons.org/licenses/by/4.0/. 


\section{Introduction}

Immune checkpoint inhibitors (ICIs) for cancer treatment have proven to be a great breakthrough in the past decade. In non-small cell lung cancer (NSCLC), ICIs have achieved convincing efficacy and tolerable safety in single-agent therapies or combined treatments and significantly prolonged the overall survival (OS) of patients (Leighl et al. 2019; Reck et al. 2019; Mok et al. 2019; Ready et al. 2019; Horn et al. 2017). During trials evaluating ICIs, several biomarkers have been found to be related to sensitivity to ICIs treatment, including high programmed death-ligand 1 (PD-L1) expression (Mok et al. 2019; Ready et al. 2019; Ott et al. 2019), a high tumor mutational burden (TMB) (Ready et al. 2019; Ott et al. 2019) and mismatch repair (MMR) deficiency (Mandal et al. 2019). In contrast, NSCLC patients harboring mutations of epidermal growth factor receptor (EGFR) and/ or anaplastic lymphoma kinase (ALK) might not benefit from ICIs treatment (Gainor et al. 2016; Haratani et al. 2017). However, the reliability of these biomarkers in predicting the prognosis of ICIs treatment in NSCLC, especially the progression-free survival (PFS) of NSCLC patients, remains unclear, and at the same time, multivariate analyses of the sensitivity to ICIs involving the biomarkers described above and the genomic signatures confirmation of patients are necessary because of the heterogeneity and high somatic mutation rate of NSCLC cells. A hazard model for the prognosis of ICIs treatment is urgently needed.

The roles of switch/sucrose nonfermenting (SWI/ SNF) chromatin remodeling complexes in a variety of biological processes during cell growth and development, including DNA replication, gene expression and cell differentiation, are essential (Wang et al. 2004; Zhang et al. 2014). These complexes are dysregulated in various cancer types (Huang et al. 2015). Canonical BRG1/BRM-associated factor (BAF), which is one of the three assembled SWI/SNF chromatin remodeling complexes (Naito et al. 2019), mainly consists of ATrich interactive domain 1A/1B (ARID1A/1B) and DPF2 subunits (Michel et al. 2018; Mashtalir et al. 2018). According to the latest studies, ARID1A deficiency may be a novel biomarker for cancer immunotherapy (Shen et al. 2018; Jiang et al. 2020), and patients with ARID1A deficiency could benefit from ICIs treatment (Okamura et al. 2020). Nevertheless, the role of ARID1B, which serves as the other subunit of ARID1 together with ARID1A, in the sensitivity to ICIs treatment is unknown. Therefore, we performed this study of a previously published cohort of patients (Rizvi et al. 2018) to clarify the role of the ARID1 subunits in the prognosis of ICIs treatment among advanced NSCLC patients and the relationships between the ARID1 subunits with the tumor immune microenvironment (TIME) or other factors related to ICIs sensitivity and to establish a prognostic model for ICIs treatment of advanced NSCLC.

\section{Methods \\ Study patients}

Using the data derived from the cBioPortal for Cancer Genomics, 240 advanced NSCLC patients who received ICIs treatment and were described in previously published work (Rizvi et al. 2018) were involved in this research. The involved patients were treated with either anti-PD-(L)1 therapy (pembrolizumab) alone or in combination with anti-cytotoxic T-cell lymphocyte-4 (antiCTLA-4) therapy (pembrolizumab+ipilimumab). Basic clinical information was collected from this cohort of patients, including age, sex and smoking history. The investigators used Response Evaluation Criteria in Solid Tumors (RECIST) version 1.1 to assess the efficacy of ICIs treatment, and PFS was assessed as the time that patients remained responsive to treatment (exhibited a complete response [CR], a partial response [PR] or stable disease [SD]). All involved patients gave their consent for the examination of targeted sequencing data, and tumor tissue samples from 86 patients were sent for PDL1 expression assessment.

\section{Survival analyses and nomogram construction}

The Kaplan-Meier plot (KM plot) method was used for univariate analyses of the involved patients, and the logrank tests were used to detect significant differences. In addition, an online tool was used to perform survival analyses for all NSCLC patients (Gyorffy et al. 2013). Variables selected by the univariate analyses were used for the construction of a nomogram. The "rms" package of R software version 3.1.2 (The R Foundation for Statistical Computing, Vienna, Austria) was used to construct the nomogram. Harrell's C-indexes ranging from 0.5 (no discrimination) to 1 (perfect discrimination) were used for the verification of discrimination (Bandos et al. 2009), and a visual calibration plot was used for the verification of calibration (Jin et al. 2017). Bootstrap analyses with 1000 resamples were used for these analyses.

\section{Bioinformatic and statistical analyses}

TMB assessment was conducted during the published clinical trial (Rizvi et al. 2018), and the exploration of the relationships between ARID1 subunits and immunocytes derived from the TIME was carried out with an online tool ( $\mathrm{Ru}$ et al. 2019). All statistical analyses were conducted by GraphPad Prism 8.0 software (GraphPad, La Jolla, CA), and Student's t tests were used to determine statistical significance. $P$ values were determined 


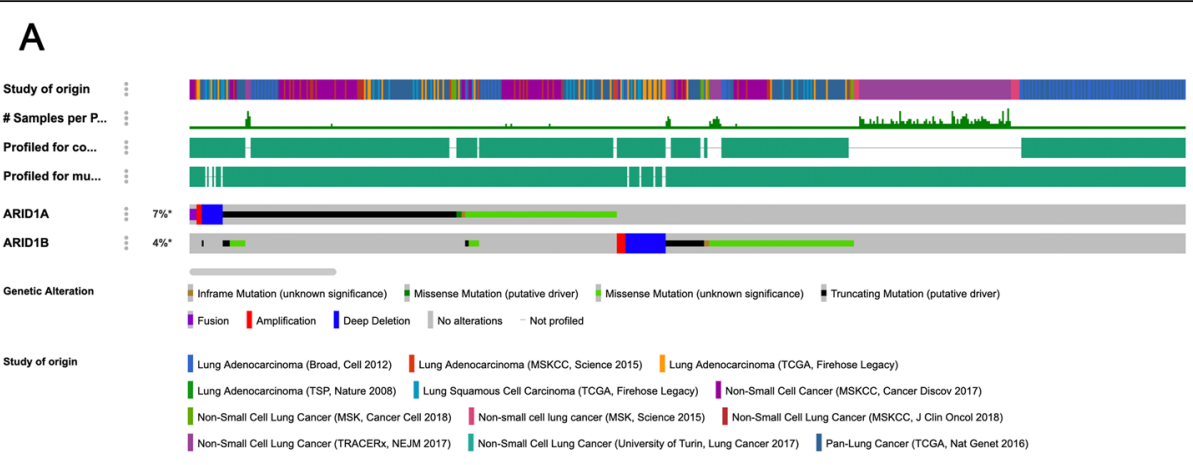

B

ARID1A

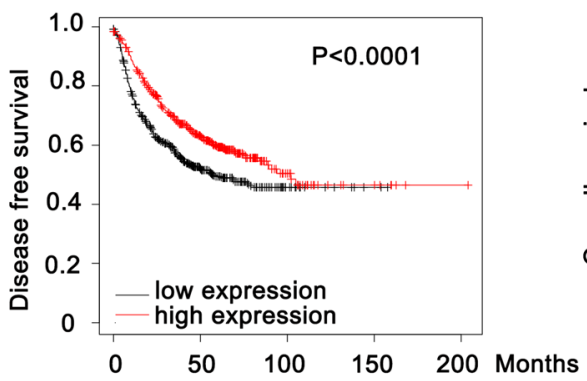

C

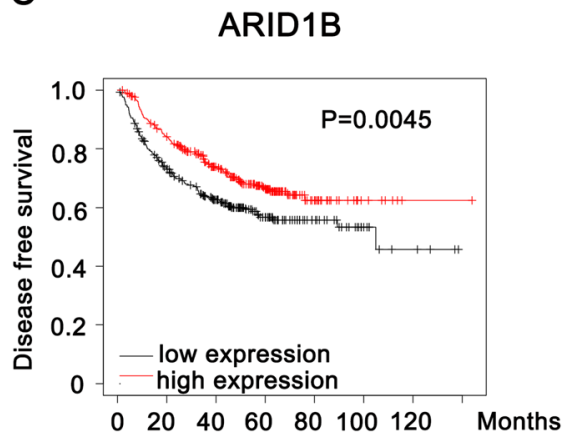

ARID1A

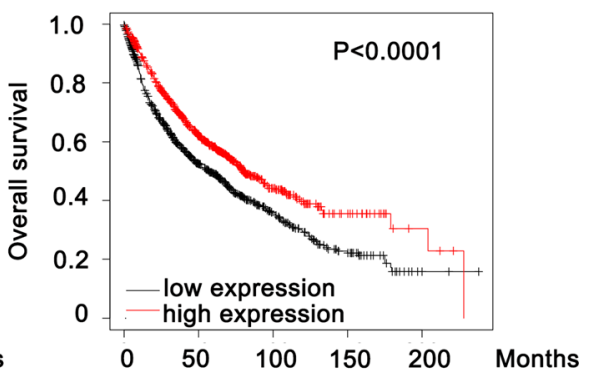

ARID1B

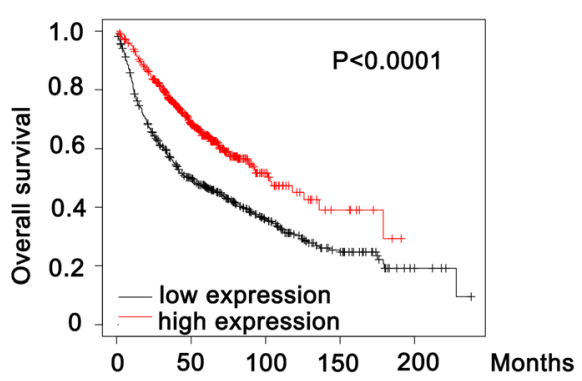

Fig. 1 ARID1 subunits are tightly associated with the prognosis of NSCLC. a. The prevalence of ARID1 subunits mutations in NSCLC patients according to the cBioPortal for Cancer Genomics; $\mathbf{b}$. The relationship between ARID1A expression and the prognosis of NSCLC patients; $\mathbf{c}$. The relationship between ARID1B expression and the prognosis of NSCLC patients

by two-tailed tests, and $P<0.05$ was considered statistically significant.

\section{Results}

The prevalence of mutant ARID1 and its role in the prognosis of NSCLC

According to the datasets acquired from the cBioPortal for Cancer Genomics, ARID1 mutation is common among NSCLC patients. As shown in Fig. 1a, the mutation frequencies of the subunits of ARID1 including $A R I D 1 A$ and $A R I D 1 B$ were 7 and $4 \%$ in NSCLC patients, respectively. As far as we are concerned, gene mutations or hypermethylation lead to low ARID1 protein expression (Guan et al. 2011; Zhang et al. 2013), therefore, we further investigated the relationship between the survival of NSCLC patients and the expression of the ARID1 protein. Figure $1 \mathrm{~b}$ and $\mathrm{c}$ describe the relationships between the disease-free survival (DFS) or OS of NSCLC patients and the expression of ARID1A or ARID1B. As shown in the figure, ARID1A and ARID1B both are convincing biomarkers for NSCLC prognosis with compelling efficiency, and ARID1A or ARID1B deficiency was 
significantly related to the poor prognosis of NSCLC (ARID1A [DFS: $P<0.0001$; OS: $P<0.0001$ ]; ARID1B [DFS: $P=0.0045$; OS: $P<0.0001]$ ).

\section{ARID1A or ARID1B mutation correlates with an improved} outcome for ICls treatment

The relationship between ARID1A or ARID1B mutation and the outcome of ICIs treatment was then studied. Through systematic analyses of the datasets from the
cBioPortal for Cancer Genomics, we found that both $A R I D 1 A$ and ARID1B mutations were associated with an improved outcome for ICIs treatment in advanced NSCLC patients. As shown in Fig. 2a, more responders $(\mathrm{CR}+\mathrm{PR}+\mathrm{SD})$ were confirmed in the mutant-type (MT) group than in the wild-type (WT) group for patients harboring ARID1A (50\% versus $19 \%, P=0.045$ ) or $A R I D 1 B(50 \%$ versus $16 \%, P=0.034$ ) mutations. Figure $2 b$ displays the median PFS (mPFS) with ICIs treatment for

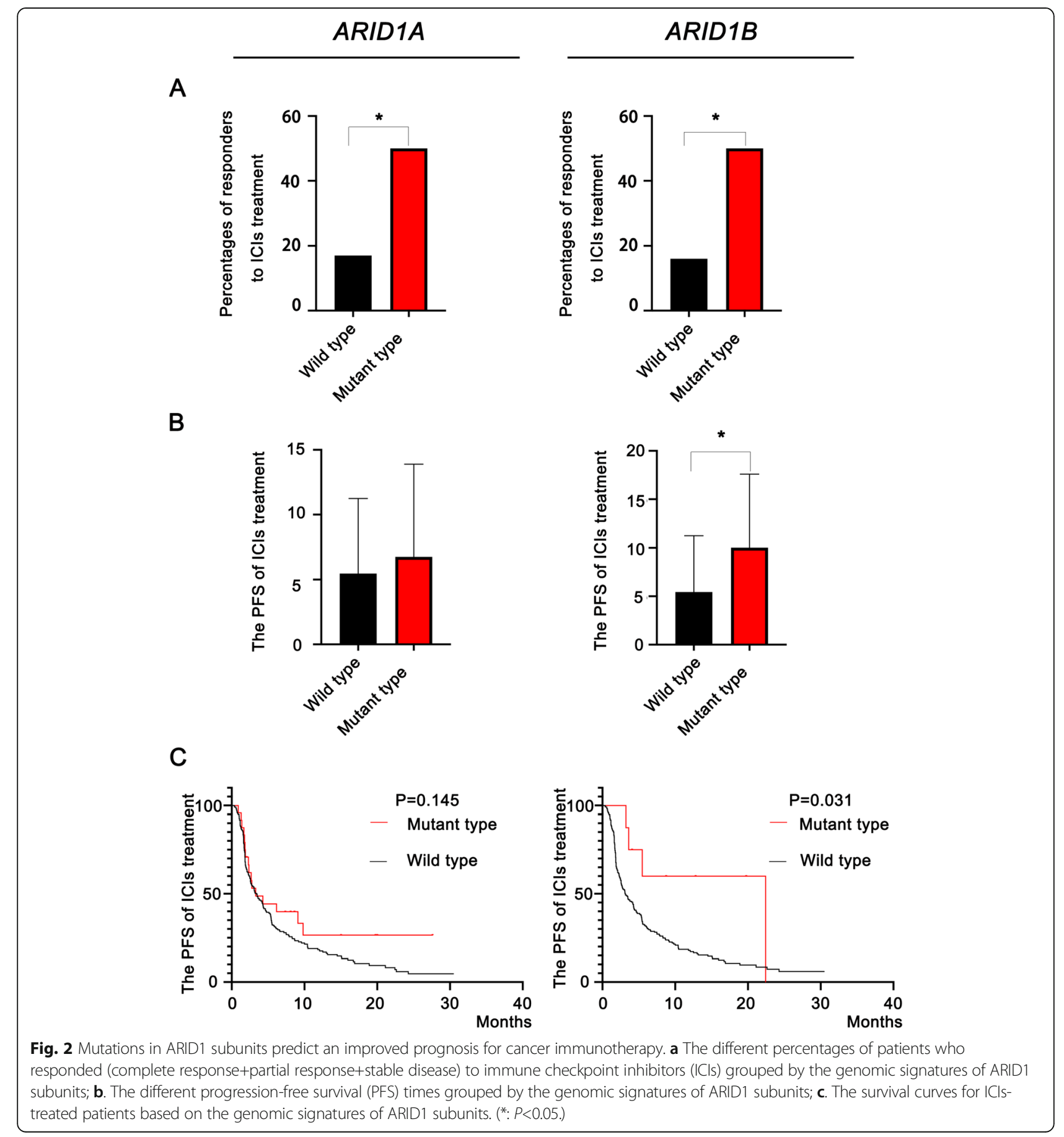


the two groups. Patients harboring ARID1A (6.8 months versus 5.5 months, $P=0.313$ ) or ARID1B (10.0 months versus 5.4 months, $P=0.032$ ) mutations benefited more from treatment and achieved a longer PFS time than those in the WT group. Survival analyses for ICIs treatment were then performed as shown in Fig. 2c. Compared with those in the WT group, patients harboring mutant $A R I D 1 B$ achieved significant survival benefits with treatment $(P=0.031)$. However, although a trend toward a difference existed for the survival curve of patients harboring mutant ARIDIA, no statistical significance was found $(P=0.145)$.

\section{Establishment of a prognostic nomogram for the prognosis of ICls treatment in NSCLC}

Eighty-six patients of the selected NSCLC cohort with integrated information on clinical features, targeted sequencing and PD-L1 expression evaluated by immunohistochemistry (IHC) were involved in the construction of the novel nomogram. First, univariate analyses were performed to identify variables to include in nomogram construction. As shown in Fig. 3a to f, multiple variables were confirmed to be significantly associated with the prognosis of ICI treatment, including EGFR mutation $(P=0.021)$, ARID1B mutation $(P=0.024)$, PD-L1 expression $(P=0.010)$, TMB $(P=0.012)$, treatment lines $(P=$ $0.003)$ and smoking history $(P=0.007)$. Through the univariate analyses, we found that patients with mutant $A R I D 1 B$, elevated PD-L1 expression ( $\geq 50 \%$ percentage positive staining), a high TMB value ( $\geq 75$ th percentage) or a history of smoking could benefit from ICIs treatment, while patients harboring mutant EGFR might not derive survival benefits from ICIs treatment. In addition, first-line administration of ICIs in advanced NSCLC patients might be a better choice than later administration. The nomogram based on these variables was then established as shown in Fig. 3g. In total, 3 types of patient information, including the clinical information, pathological information and genomic signatures, were included in the nomogram. Through this novel nomogram, physicians could easily obtain a score based on the Cox regression model for each variable listed in the graph, and then the total number would be assessed as the sum of all variable scores. Therefore, the survival risks of ICIs treatment for advanced NSCLC patients could be quantified before treatment. The $\mathrm{C}$-index for this prognostic model was 0.71 , which suggests that the model has a relatively robust ability to predict the PFS of advanced NSCLC patients treated with ICIs. The calibration plots shown in Fig. 3h indicated that the probabilities of our prognostic model agreed with the accuracy probabilities on acceptable scales (the dashed lines in the calibration plots correspond to a $10 \%$ margin of error).
The mutation status of $A R I D 1 A$ or $A R I D 1 B$ is associated with the TMB level, PD-L1 expression and the TIME modulation of NSCLC

Based on the research above, we reasonably deduced that ARID1A or ARID1B mutation serves as a novel biomarker for ICIs treatment and could have a connection with factors that are proven to be associated with sensitivity to cancer immunotherapy. As shown in Fig. 4a, ARID1A or ARID1B mutations were associated with a higher TMB value (ARID1A: 16.2 versus 9.3, $P=0.001$; $A R I D 1 B: 17.1$ versus 9.4, $P=0.020)$ and a higher proportion of PD-L1-positive cells (ARID1A: $38.9 \%$ versus $12.9 \%, P=0.040 ; A R I D 1 B: 41.3 \%$ versus $12.4 \%, \mathrm{P}=0.020$ ) in advanced NSCLC patients. Figure $4 \mathrm{~b}$ reveals the relationships between the TIME with the expression of ARID1A or ARID1B. As shown in the figure, ARID1A or ARID1B expression was related to immunosuppression in 517 lung adenocarcinoma (LUAD) samples and 501 lung squamous cell carcinoma (LUSC) samples, which is mainly characterized by significant reductions in the abundances of activated CD8+ $\mathrm{T}$ cells and activated dendritic cells (DCs). This result suggested that ARID1A or ARID1B deficiency might modulate the TIME via the activation of the antigen presentation process and cellular immunity and thus contribute to the change in the sensitivity to ICIs treatment.

\section{Discussion}

As is well known, the PD-L1 expression or TMB level did not disclose satisfied efficiency in selecting patients who might benefit from immunotherapy while plasma NGS circulating-free DNA (cfDNA) analysis in NSCLC might provides new biomarkers for cancer immunotherapy (Rossi et al. 2020). According to the previous studies, the co-mutations of TP53 and STK11 serve as an important role in modulating the TIME of NSCLC and related to the sensitivity to ICIs treatment (Skoulidis and Heymach 2019). Especially for STK11 and KEAP1 mutations, which were found more prevalent altered in $K R A S$-mutated LUAD, demonstrate their roles in inducing the primary resistance to ICIs treatment (Skoulidis and Heymach 2019; Skoulidis et al. 2018; PapillonCavanagh et al. 2020). On the contrary, mutations of ARID1A, which belongs to the ARID1 family member, might be associated with a different outcome among patients received ICIs treatment and participate to the improved ICIs outcome in NSCLC via associating KRAS mutations as reported by Gandara D, et al. Besides, Goswami $\mathrm{S}$, et al. confirmed that ARID1A mutations plus CXCL13 expression as combinatorial biomarkers to predict the sensitive phenotype to ICIs in metastatic urothelial carcinoma (Goswami et al. 2020). In addition, the NGS examination based on MYSTIC trial (Rizvi et al. 2020) revealed that ARID1A mutations are associated 

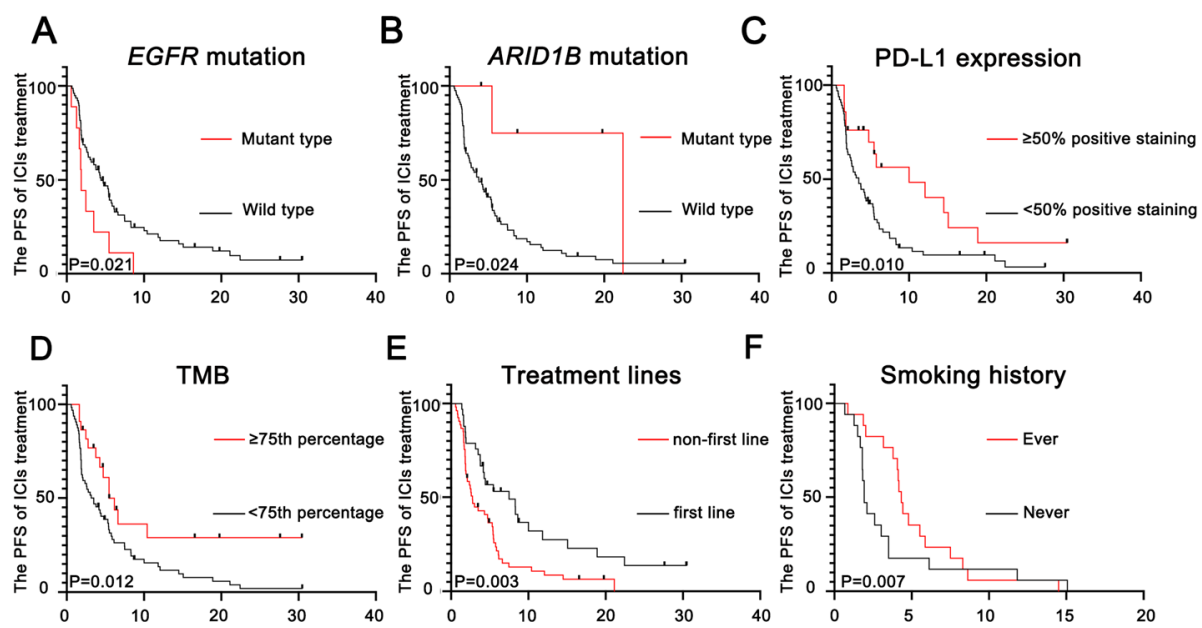

G

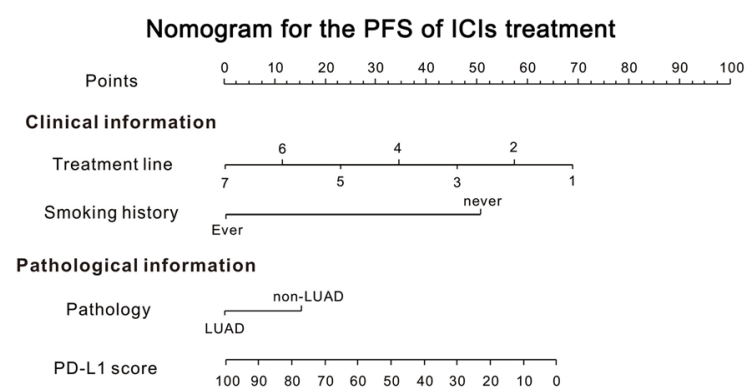

Genomic signatures

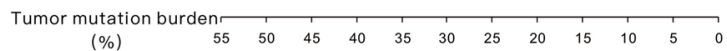

ARID1B mutation ${ }_{\text {mutant type }}$ wild-type

Prognosis of ICIs treatment

$$
\begin{aligned}
& \begin{array}{lllllllllllll}
\text { Total Points } & 0 & 50 & 100 & 150 & 200 & 250 & 300 & 350 & 400 & 450 & 500 & 550
\end{array} \\
& \text { 6-month PFS } \quad \begin{array}{lllllllll} 
& 0.85 & 0.70 & 0.5 & 0.3 & 0.1 \\
\cline { 2 - 4 } & 0.95 & 0.80 & 0.6 & 0.4 & 0.2
\end{array} \\
& \text { 12-month PFS } \\
& \begin{array}{lllllll}
0.85 & 0.70 & 0.5 & 0.3 & 0.1
\end{array} \\
& \text { 18-month PFS } \quad \begin{array}{cccccc}
0.80 & 0.6 & 0.4 & 0.2 \\
0.85 & 0.70 & 0.5 & 0.3 & 0.1
\end{array}
\end{aligned}
$$

H Calibration plot

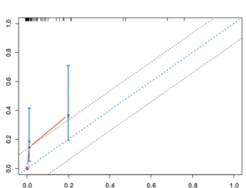

Fig. 3 Novel nomogram to predict the prognosis of immune checkpoint inhibitor (ICI) treatment. a The survival curves for ICls-treated patients based on the EGFR mutational status; b. The survival curves for ICls-treated patients based on the ARID1B mutational status; $\mathbf{c}$. The survival curves for ICls-treated patients based on PD-L1 expression; $\mathbf{d}$. The survival curves for ICls-treated patients based on the tumor mutational burden (TMB); e. The survival curves for ICls-treated patients based on treatment lines; f. The survival curves for ICls-treated patients based on smoking history; $\mathbf{g}$. The novel nomogram based on patient information to predict the prognosis of $\mathrm{ICI}$ treatment; $\mathbf{h}$. The calibration plot for the nomogram

with improved outcomes with the PD-1 inhibitor durvalumab plus the CTLA4 inhibitor tremelimumab reported by Rizvi NA, et al. Based on these studies for new biomarkers of cancer immunotherapy especially for ARID1A mutations, we conducted our research in order to further investigate the significance of ARID1 mutations, including $A R I D 1 A$ and $A R I D 1 B$, in predicting the prognosis of ICIs treatment for advanced NSCLC.
To the best of our knowledge, our research is the first to illustrate the roles of the ARID1 subunits in the prognosis of cancer immunotherapy and to establish a prognostic model for immunotherapy in advanced NSCLC patients. Through this research, the roles of ARID1A and ARID1B in the prognosis of NSCLC were clearly clarified, and both ARID1A deficiency and ARID1B deficiency were related to the poor prognosis of NSCLC 


\section{A}

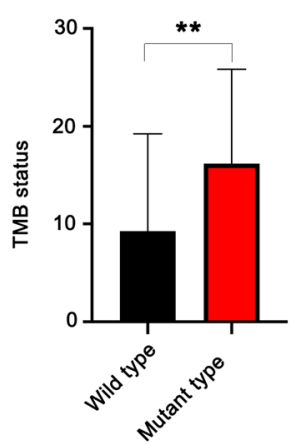

B

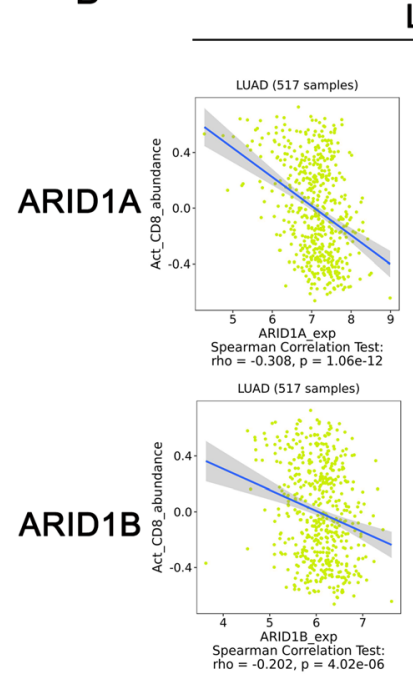

C

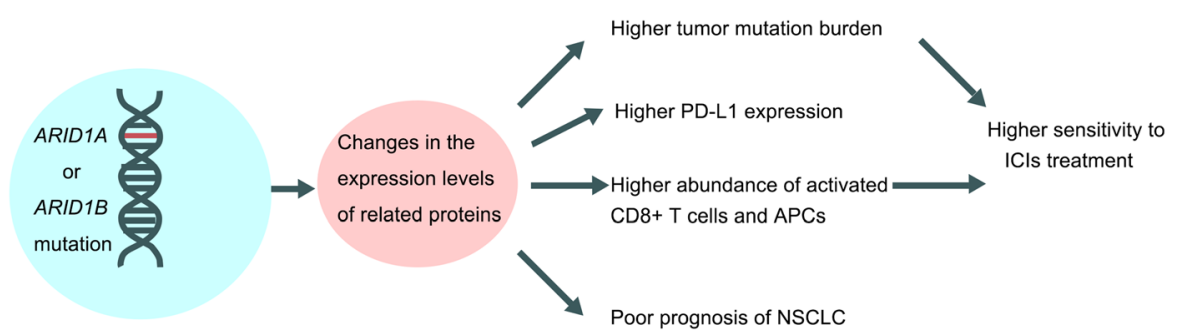

Fig. 4 ARIDIA or ARIDIB mutations are tightly associated with sensitivity to immune checkpoint inhibitors (ICls). a. The comparison of tumor mutational burden (TMB) values and PD-L1 expression grouped by the genomic signatures of ARID1 subunits; $\mathbf{b}$. The correlations between ARID1A or ARID1B expression and the abundances of activated CD8+ T cells and activated dendritic cells (DC) in lung adenocarcinoma (LUAD) and lung squamous cell carcinoma (LUSC); . The underlying relationships between prognosis and ARIDIA or ARID1B mutation deduced from this research. $(* *: P<0.01 ; *$ : $P<0.05)$

patients. However, ARID1A or ARID1B mutations and the resultant functional deficiencies were tightly associated with the sensitive phenotype for cancer immunotherapy. Patients harboring ARID1A or ARID1B mutations were more likely to have a good response to treatment with ICIs, and their PFS time might be prolonged through ICIs treatment. These correlations were also verified by the prognostic nomogram based on the Cox regression model established in this research. Here, we provided patients and physicians with this novel nomogram to estimate the survival risks of ICI treatment and determine an appropriate regimen for treatment and follow-up before treatment is initiated.

The results from the latest studies suggest that ARID1A mutation and the resultant functional deficiency are associated with the elevation in PD-L1 expression and the TMB value in ovarian cancer (Shen et al. 2018) and gastric cancer (Li et al. 2019), which indicates the underlying relationship between ARID1A and potential therapeutic anticancer immunity. Whether this 
correlation can be verified in NSCLC and the potential role of ARID1B in cancer immunotherapy remain to be further studied. The bioinformatic analyses in this research indicated that ARID1A or ARID1B mutations were associated with instability in the cancer cell genome and cancer mutability, as indicated by the elevated $\mathrm{TMB}$, which is a proven biomarker for cancer immunotherapy. Patients harboring ARID1A or ARID1B mutations are likely to exhibit enhanced expression of PD-L1. In addition, through the activation of the antigen presentation process and anticancer cellular immunity, ARID1A and ARID1B deficiencies could modulate the TIME of NSCLC and initiate a therapeutic immune reaction to tumors. In this research, we confirmed the important role of ARID1A deficiency in elevating cancerous mutability and changing tumors to exhibit an aggressive phenotype in NSCLC, which was proposed by published works (Shen et al. 2018; Jiang et al. 2020; Okamura et al. 2020; Rossi et al. 2020) on other cancer types. Additionally, our research is the first to investigate the role of ARID1B, which is another ARID1 subunit similar to ARID1A, in changing the phenotype of cancer. Given the results reported in this research, we proposed that ARID1A and ARID1B might be equally important in cancer immunotherapy and the prognosis of NSCLC.

As shown in Fig. 4c, we generated new results in this research and reasonably deduced that mutation of ARID1A or ARID1B leads to changes in the expression levels of related proteins, which results in the poor prognosis of NSCLC on the one hand but enhances the sensitivity to ICI treatment in advanced NSCLC through elevating the TMB, enhancing PD-L1 expression and modulating the anticancer immune reaction on the other hand. Admittedly, our research might have several limitations. Further investigations into the role of ARID1B in cancer immunotherapy are needed, given the small sample size in this research. Although the results suggest that ARID1A and ARID1B may participate in the modulation of the TIME and are associated with the elevations in tumor mutability and PD-L1 expression, no molecular mechanism was explored in this research. Further studies are needed to explore the mechanism underlying these correlations.

\section{Conclusion}

ARID1A and ARID1B could serve as novel biomarkers for the prognosis and sensitivity to ICIs treatment of advanced NSCLC. ARID1A or ARID1B mutations and the resultant functional deficiencies are tightly associated with cancer mutability, PD-L1 expression and TIME modulation and are associated with a good prognosis for ICIs treatment. The relationship between the prognosis of ICIs treatment and ARIDIB mutation could be verified by the novel nomogram for ICIs treatment.

\section{Abbreviations}

SWI/SNF: Switch/sucrose nonfermenting; ARID1A: AT-rich interactive domain 1A; ARID1B: AT-rich interactive domain 1B; BAF: BRM-associated factor; NSCL C: Non-small cell lung cancer; EGFR: Epidermal growth factor receptor;

ALK: Anaplastic lymphoma kinase; IHC: Immunohistochemistry; DFS: Diseasefree survival; ICls: Immune checkpoint inhibitors; PD-L1: Programmed deathligand 1; TMB: Tumor mutational burden

\section{Acknowledgements}

Our work was supported by Special Funding for the Qilu Sanitation and Health Leading Talents Cultivation Project (to Helei Hou); the Chinese Postdoctoral Science Foundation (2017 M622143 to Helei Hou); and the Qingdao Postdoctoral Application Research Funded Project (2016052 to Helei Hou).

\section{Authors' contributions}

Conception/design: Helei Hou and Hong Li; Provision of study material or patients: Helei Hou and Dantong Sun; Collection and/or assembly of data: Dantong Sun, Lu Tian, Yang Wo and Qiaoling Liu; Data analysis and interpretation: Dantong Sun, Lu Tian; Manuscript writing: Helei Hou, Dantong Sun and Yan Zhu; Final approval of manuscript: All authors.

\section{Funding}

Special Funding for the Qilu Sanitation and Health Leading Talents Cultivation Project (to Helei Hou); the Chinese Postdoctoral Science Foundation (2017 M622143 to Helei Hou); and the Qingdao Postdoctoral Application Research Funded Project (2016052 to Helei Hou).

\section{Availability of data and materials}

All data generated during this study are included in this published article. The datasets generated in the current study are available in the cBioportal for Cancer Genomics [Cerami et al. 2012; Gao et al. 2013] (http://www. cbioportal.org/).

\section{Ethics approval and consent to participate}

Not applicable.

\section{Consent for publication}

Not applicable.

\section{Competing interests}

The authors declare no conflicts of interest.

\section{Author details}

${ }^{1}$ Precision Medicine Center of Oncology, the Affiliated Hospital of Qingdao University, 59 Haier Road, Qingdao 266000, Shandong, China. ${ }^{2}$ College of Environmental Science and Engineering, Ocean University of China, Qingdao 266100, China. ${ }^{3}$ Department of Medical Oncology, the Municipal Hospital of Qingdao, Qingdao 266000, China. ${ }^{4}$ Department of Thoracic Surgery, the Affiliated Hospital of Qingdao University, Qingdao 266000, Shandong, China. ${ }^{5}$ Department of Medical Oncology, Qingdao West Coast New Area Central Hospital, Qingdao 266555, China. ${ }^{6}$ Medical Animal Laboratory, the Affiliated Hospital of Qingdao University, Qingdao 266000, Shandong, China. ${ }^{7}$ State Key Laboratory of Oncogenes and Related Genes, Shanghai Cancer Institute, Renji Hospital, Shanghai Jiaotong University School of Medicine, Shanghai 200032, China.

Received: 30 May 2020 Accepted: 3 August 2020

Published online: 13 August 2020

\section{References}

Cerami E, Gao J, Dogrusoz U, et al. The cBio cancer genomics portal: an open platform for exploring multidimensional cancer genomics data. Cancer Discov. 2012;2(5):401-4.

Bandos Al, Rockette HE, Song T, et al. Area under the free-response ROC curve (FROC) and a related summary index. Biometrics. 2009;65(1):247-56.

Gainor JF, Shaw AT, Sequist LV, et al. EGFR mutations and ALK rearrangements are associated with low response rates to PD-1 pathway blockade in nonsmall cell lung cancer: a retrospective analysis. Clin Cancer Res. 2016;22(18): 4585-93. 
Gao J, Aksoy BA, Dogrusoz U, et al. Integrative analysis of complex cancer genomics and clinical profiles using the cBioPortal. Sci Signal. 2013;6(269):11.

Goswami S, Chen Y, Anandhan S, et al. ARID1A mutation plus CXCL13 expression act as combinatorial biomarkers to predict responses to immune checkpoint therapy in mUCC. Sci Transl Med. 2020;12(548):eabc4220.

Guan B, Mao TL, Panuganti PK, et al. Mutation and loss of expression of ARID1A in uterine low-grade endometrioid carcinoma. Am J Surg Pathol. 2011;35(5): 625-32.

Gyorffy B, Surowiak P, Budczies J, et al. Online survival analysis software to assess the prognostic value of biomarkers using transcriptomic data in non-smallcell lung cancer. PLoS One. 2013;8(12):e82241.

Haratani $\mathrm{K}$, Hayashi $\mathrm{H}$, Tanaka $\mathrm{T}$, et al. Tumor immune microenvironment and nivolumab efficacy in EGFR mutation-positive non-small-cell lung cancer based on T790M status after disease progression during EGFR-TKI treatment. Ann Oncol. 2017:28(7):1532-9.

Horn L, Spigel DR, Vokes EE, et al. Nivolumab versus docetaxel in previously treated patients with advanced non-small-cell lung cancer: two-year outcomes from two randomized, open-label, phase III trials (CheckMate 017 and CheckMate 057). J Clin Oncol. 2017;35(35):3924-33.

Huang HT, Chen SM, Pan LB, et al. Loss of function of SWI/SNF chromatin remodeling genes leads to genome instability of human lung cancer. Oncol Rep. 2015:33(1):283-91.

Jiang T, Chen X, Su C, et al. Pan-cancer analysis of ARID1A alterations as biomarkers for immunotherapy outcomes. J Cancer. 2020;11(4):776-80.

Jin C, Cao J, Cai Y, et al. A nomogram for predicting the risk of invasive pulmonary adenocarcinoma for patients with solitary peripheral subsolid nodules. J Thorac Cardiovasc Surg. 2017;153(2):462-9.

Leighl NB, Hellmann MD, Hui R, et al. Pembrolizumab in patients with advanced non-small-cell lung cancer (KEYNOTE-001): 3-year results from an open-label, phase 1 study. Lancet Respir Med. 2019;7(4):347-57.

Li L, Li M, Jiang Z, et al. ARID1A mutations are associated with increased immune activity in gastrointestinal cancer. Cells. 2019;8(7).

Mandal R, Samstein RM, Lee KW, et al. Genetic diversity of tumors with mismatch repair deficiency influences anti-PD-1 immunotherapy response. Science. 2019;364(6439):485-91.

Mashtalir N, D'Avino AR, Michel BC, et al. Modular organization and assembly of SWI/SNF family chromatin remodeling complexes. Cell. 2018;175(5):1272-88.

Michel BC, D'Avino AR, Cassel SH, et al. A non-canonical SWl:SNF complex is a synthetic lethal target in cancers driven by BAF complex perturbation. Nat Cell Biol. 2018:20(12):1410-20.

Mok TSK, Wu YL, Kudaba I, et al. Pembrolizumab versus chemotherapy for previously untreated, PD-L1-expressing, locally advanced or metastatic nonsmall-cell lung cancer (KEYNOTE-042): a randomised, open-label, controlled, phase 3 trial. Lancet. 2019;393(10183):1819-30.

Naito T, Udagawa H, Umemura S, et al. Non-small cell lung cancer with loss of expression of the SWI/SNF complex is associated with aggressive clinicopathological features, PD-L1-positive status, and high tumor mutation burden. Lung Cancer. 2019;138:35-42.

Okamura R, Kato S, Lee S, et al. ARID1A alterations function as a biomarker for longer progression-free survival after anti-PD-1/PD-L1 immunotherapy. J Immunother Cancer. 2020:8(1):e000438.

Ott PA, Bang YJ, Piha-Paul SA, et al. T-cell-inflamed gene-expression profile, programmed death ligand 1 expression, and tumor mutational burden predict efficacy in patients treated with pembrolizumab across 20 cancers: KEYNOTE-028. J Clin Oncol. 2019;37(4):318-27.

Papillon-Cavanagh S, Doshi P, Dobrin R, et al. STK11 and KEAP1 mutations as prognostic biomarkers in an observational real-world lung adenocarcinoma cohort. ESMO Open. 2020;5(2):e000706.

Ready N, Hellmann MD, Awad MM, et al. First-line nivolumab plus ipilimumab in advanced non-small-cell lung cancer (CheckMate 568): outcomes by programmed death ligand 1 and tumor mutational burden as biomarkers. $J$ Clin Oncol. 2019;37(12):992-1000.

Reck M, Rodríguez-Abreu D, Robinson AG, et al. Updated analysis of KEYNOTE024: pembrolizumab versus platinum-based chemotherapy for advanced non-small-cell lung cancer with PD-L1 tumor proportion score of 50\% or greater. J Clin Oncol. 2019;37(7):537-46.

Rizvi H, Sanchez-Vega F, La K, et al. Molecular determinants of response to antiprogrammed cell death (PD)-1 and anti-programmed death-ligand 1 (PD-L1) blockade in patients with non-small-cell lung cancer profiled with targeted next-generation sequencing. J Clin Oncol. 2018;36(7):633-41.
Rizvi NA, Cho BC, Reinmuth N, et al. Durvalumab with or without tremelimumab vs standard chemotherapy in first-line treatment of metastatic non-small cell lung cancer: the MYSTIC phase 3 randomized clinical trial. JAMA Oncol. 2020; 6(5):661-74.

Rossi G, Russo A, Tagliamento M, et al. Precision medicine for NSCLC in the era of immunotherapy: new biomarkers to select the most suitable treatment or the most suitable patient. Cancers (Basel). 2020;12(5):1125.

Ru B, Wong CN, Tong Y, et al. TISIDB: an integrated repository portal for tumorimmune system interactions. Bioinformatics. 2019;35(20):4200-2.

Shen J, Ju Z, Zhao W, et al. ARID1A deficiency promotes mutability and potentiates therapeutic antitumor immunity unleashed by immune checkpoint blockade. Nat Med. 2018;24(5):556-62.

Skoulidis F, Goldberg ME, Greenawalt DM, et al. STK11/LKB1 mutations and PD-1 inhibitor resistance in KRAS-mutant lung adenocarcinoma. Cancer Discov. 2018:8(7):822-35.

Skoulidis F, Heymach JV. Co-occurring genomic alterations in non-small-cell lung cancer biology and therapy. Nat Rev Cancer. 2019;19(9):495-509.

Wang X, Nagl NG, Wilsker D, et al. Two related ARID family proteins are alternative subunits of human SWI/SNF complexes. Biochem J. 2004;383(Pt 2):319-25.

Zhang X, Sun Q, Shan M, et al. Promoter hypermethylation of ARID1A gene is responsible for its low mRNA expression in many invasive breast cancers. PLoS One. 2013;8(1):e53931.

Zhang $Y, X u X$, Zhang $M$, et al. ARID1A is downregulated in non-small cell lung cancer and regulates cell proliferation and apoptosis. Tumour Biol. 2014;35(6): 5701-7.

\section{Publisher's Note}

Springer Nature remains neutral with regard to jurisdictional claims in published maps and institutional affiliations.

Ready to submit your research? Choose BMC and benefit from:

- fast, convenient online submission

- thorough peer review by experienced researchers in your field

- rapid publication on acceptance

- support for research data, including large and complex data types

- gold Open Access which fosters wider collaboration and increased citations

- maximum visibility for your research: over $100 \mathrm{M}$ website views per year

At $\mathrm{BMC}$, research is always in progress.

Learn more biomedcentral.com/submissions 